\title{
Open Ecosystems, Collaborative Networks and Service Entities Integrated Modeling Approach
}

\author{
Rubén Darío Franco, Angel Ortiz Bas, Pedro Gómez-Gasquet, \\ and Raúl Rodriguez Rodriguez \\ Research Centre on Production Management and Engineering, \\ Ciudad Politécnica de la Innovación, Edificio 8B, Acceso L, \\ Pta 246022 Valencia, Spain \\ \{dfranco, aortiz, pgomez, raurodro\} @cigip.upv.es
}

\begin{abstract}
This paper introduces an integrated modeling approach that has been used to design and implement the ColNet platform. ColNet is the result of creating an integrated system, supporting distributed business processes design and execution in a Collaborative Network $(\mathrm{CN})$ belonging to an Open Ecosystem (VBE). The work has been carried out as a part of the FP7 REMPLANET European Project, which aims at providing methods and tools for better decision-making in non-hierarchical collaborative networks. The functional alignment of Open Service Ecosystems, Collaborative Networks and Services Entities lifecycles is adopted as main functional requirements for the platform design and implementation while, at the same time, they raise an integrated modeling need. Both constitute the underlying approach followed when designing and implementing ColNet and are briefly described here.
\end{abstract}

Keywords: VBE modeling, CN Management System, Service Entities.

\section{Introduction}

The scientific community related to Collaborative Networked Organizations (CNO) research has agreed on the usefulness and convenience of considering Virtual organizations Breeding Environments (VBE) as fertile spaces where CN lifecycle takes place, from its creation to its final dissolution. Similar considerations have been stated for Open Service Ecosystems [1]. In this work, both terms are used interchangeably as in [1].

Broadly speaking, VBE are expected to establish normative rules, define reference processes and to host a set of registered nodes which, in turn, will be selected to arrange, setup and launching a new $\mathrm{CN}$.

Two research streams have been heavily developed, and contributed, in the past, from both $\mathrm{VBE}$ and $\mathrm{CN}$ management perspectives. Moreover, in the ECOLEAD project [2], VBE [3] and $\mathrm{CN}$ [4] management systems were characterized and functionally described but interactions among both systems were just briefly mentioned.

Based on such previous works, what this paper addresses: 
Is it possible to design and implement a single IT system managing, in an integrated way, both VBE and CN operations? If so, which architectural principles must be considered?

Service Entities have been proposed [5], [6] as a third component of the model which would play a main role in supporting the engineering and operating of those $\mathrm{CN}$ in a VBE, based on a service-oriented approach.

This paper aims at contributing in that integration by means of two mechanisms:

- The functional alignment of the three main lifecycles, namely Ecosystem (or $\mathrm{VBE}$ ), CN and Service Entities respectively, and

- The definition and identification of the corresponding modeling perspectives as architectural support of an integrated management system.

The rest of the paper has been structured as follows: Section 2 introduces a set of background concepts that are needed for better understanding the focus of this contribution. In Section 3, main processes of the above mentioned three lifecycles will briefly described. Section 4 discusses on the functional integration of those lifecycles and Section 5 shows how the integration is proposed from the alignment of their corresponding modeling views perspective. Finally, in Section 6, some conclusions and next actions are introduced.

\section{Related Concepts}

\subsection{Virtual Breeding Environments and Collaborative Networks}

Collaboration between partners is a preferred way to ensure optimal resource balance and to get perdurable benefits [7]. Time of preparation and difficulty of launching a $\mathrm{CN}$ will increase in the same proportion of the number of potential partners and complexity of collaborative processes to be carried out.

Virtual Breeding Environments [8] are aimed to harmonize the preparedness level of involved organizations while, at the same time, a collaborative infrastructure is deployed in order to deal with interoperability problems at different levels: communications, data, services, processes or business [9].

In VBEs, the main goal is to restrict the number of potential participants by drawing a border to the open universe and allowing some partners to come inside. Those partners have to agree on common operating principles: business semantics, strategies or goals, distributed business processes management practices or even common ICT tools. Rapidness and flexibility in $\mathrm{CN}$ preparation and launching are requirements that any VBE management system must accomplish.

\subsection{Service Entities}

Service Entities (SE) are proposed as modeling constructs for CNOs, for both the Structural and Functional dimensions. As they have been defined, a Service Entity [5] is the result of logically tying together: 
- A finite set of business services - understood as functional capabilities that a service provider may offer to third parties by means of public interfaces - which jointly define the expected behavior of these entities and,

- A finite set of attributes which allow characterize and distinguish among them.

There exist two different types of SE: Abstract Service Entities (ASE) and Concrete Service Entity (CSE).

- An Abstract Service Entity is a generic building block used to represent different 'types' of entities that are present in the problem domain, mainly at Ecosystem level. ASEs are not linked to any specific instance of entities they are defining. They only represent the abstract definition of the attributes and also the specifications of the business service interfaces being defined for them.

- Concrete Service Entity: CSE are expected to be real entities of the problem domain, CSE are instances of actual ASE. Instantiating an ASE means to provide meaningful values to its attributes and specific implementation for its service interfaces (i.e. bank account validation and account balance sheet). At modeling level, this means to create an open repository where CSEs can be searched, discovered and used to create and launch $\mathrm{CN}$ instances.

\section{Functional Lifecycle Integration}

The synergies of an integrated approach for VBE and $\mathrm{CN}$ management have been raised by Romero et al in [10]. In that work, a common framework for VBE and CN is proposed. Along the respective lifecycles, the authors introduce a set of business processes which provide the expected functional linkage between VBE and CN roles.

Despite this process-centric contribution, building an integrated system for VBE and $\mathrm{CN}$ will still require additional elements in order to achieve a truly operational status for managing extended business process involving also the nodes.

As a complement, this work takes advantage of the SE approach in order to realize the integration as expected. Next sections describe how this approach is also integrated with those already mentioned.

\subsection{Ecosystem Lifecycle}

The Ecosystem Manager is in charge of managing the creation; operation and dissolution of ecosystems (see Fig. 1).

First stage is aimed at setting up and running all the technical tasks supporting the creation of the ecosystem. In the ColNet platform, this task mostly deals with the initialization of databases, creation of empty repositories and main roles assignment.

Main processes of the operational phase are also represented in Fig. 1. For this work, most relevant ones are:.

- Service Entities Management: this task is related to the profiling of generic nodes (ASE) that later on will exist inside the ecosystem. 
- $\quad$ Reference Process modeling: refers to the design of business processes that include ASE as its main actors and their services as functional blocks to be included in the process model.

- Business Documents and messages: aimed at defining the actual business documents and message schemas that may rule the information exchanges and co-ordination patterns inside the ecosystem.

- Collaborative Networks Management: includes the creation of $\mathrm{CN}$ and supervising some activities related to their lifecycle.

The final stage is aimed at supporting the ecosystem dissolution that will consist of a set of activities collecting the knowledge gathered from its operational phase.

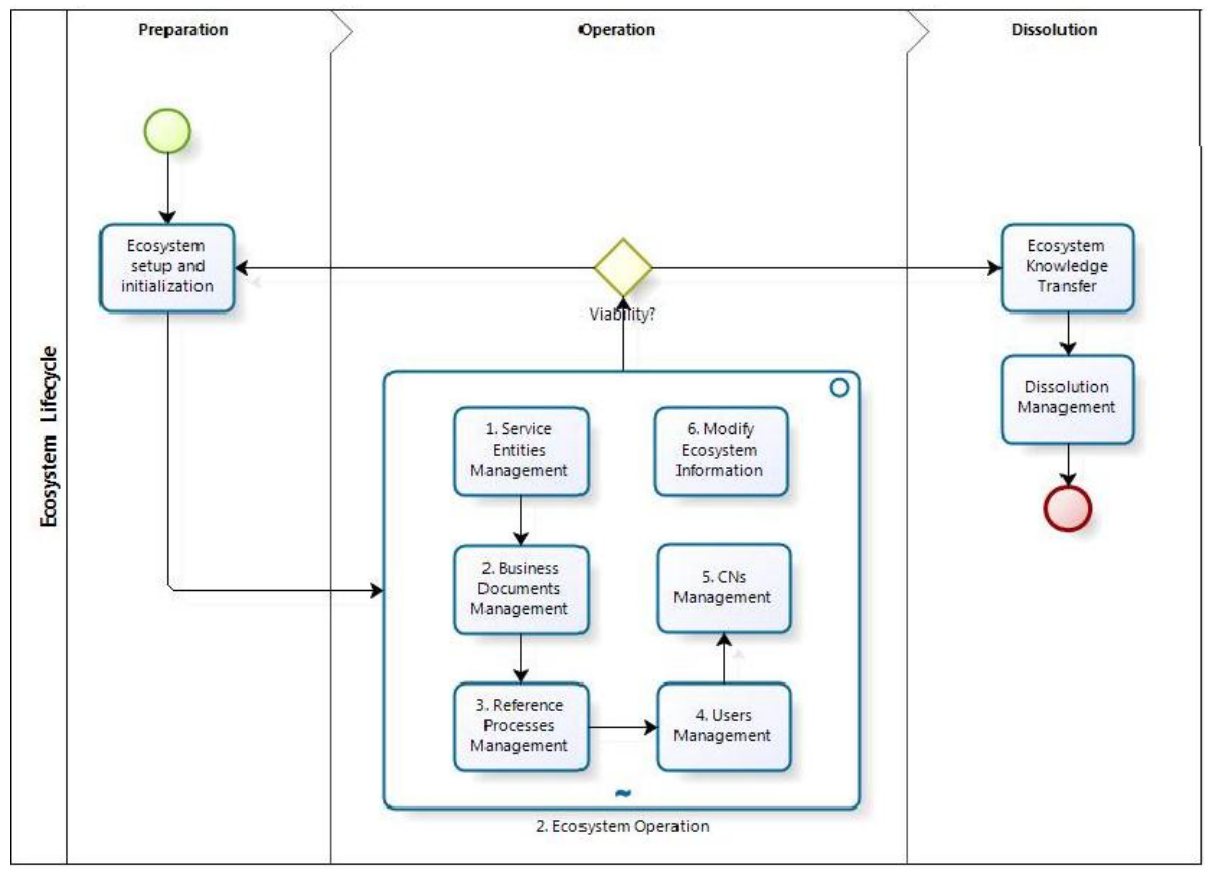

Fig. 1. Main processes at each stage of the ecosystem's lifecycle

\subsection{CN Lifecycle}

As depicted in Fig. 2, $\mathrm{CN}$ are created, operated and dissolved. For creating a new $\mathrm{Cn}$, the $\mathrm{CN}$ manager will request its approval to the ecosystem manager of the VBE to which the $\mathrm{CN}$ will belong to. Once approved, the $\mathrm{CN}$ configuration can be started.

During the operational stage of the $\mathrm{CN}$, the manager will have several duties to accomplish. Among them, it is possible to mention the management of:

- Network structure: this task implies to decide which available resources of the network will be invited to join the network for future transactions. 
- $\quad$ Concrete nodes management: interested nodes may request joining a $\mathrm{CN}$ as preferred partners. Other nodes will be invited to join the $\mathrm{CN}$.

- Network business documents and messages: actual XML schemas that are used for both information exchange and co-ordination purposes in the $\mathrm{CN}$.

- Network processes: aimed at creating the collaborative processes that will represent the operational perspective of the $\mathrm{CN}$.

- Network monitoring: ColNet also provides managers with simple monitoring tools intended to monitor main $\mathrm{CN}$ parameters.

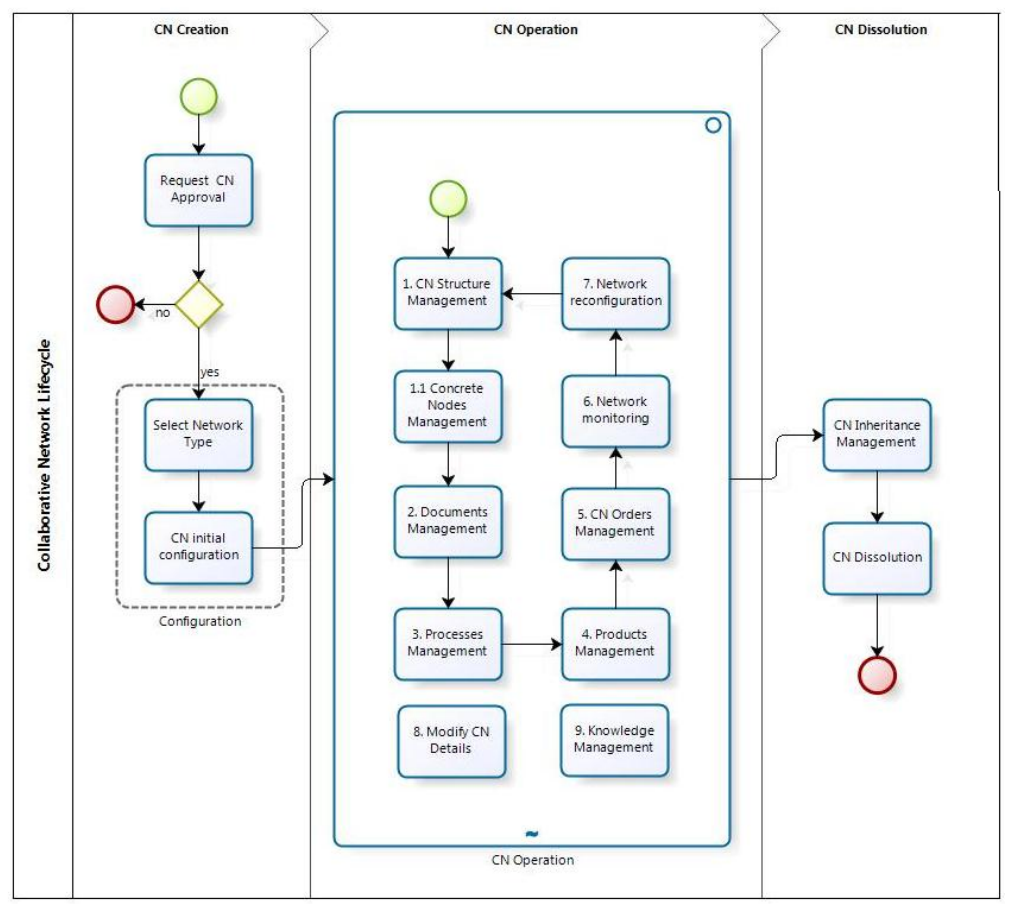

Fig. 2. Main processes at each stage of the collaborative network's lifecycle

After the $\mathrm{CN}$ has met its business goals, the manager may decide on keep it operational or proceed to its dissolution. In this case, proper mechanisms will be needed to support both the inheritance management and the final repositories disposal.

\subsection{Service Entities Lifecycle}

Each Ecosystem member is represented by a CSE instance. This role can be played by any organizational or physical resource (for instance, a whole company, a single department of that company, a person of that department, a truck of that company, etc.) being able to provide functional capabilities, from a service-oriented perspective, that will be contributing to the extended business process execution. 
As shown in Fig. 3, the Service Entities (SE) lifecycle is composed of three main stages: preparation, operation and dissolution.

At the preparation stage, the node manager will need to register it into the ecosystem. That means to identify which generic node (ASE) better describes the node being registered and creating a concrete instance (CSE) of it.

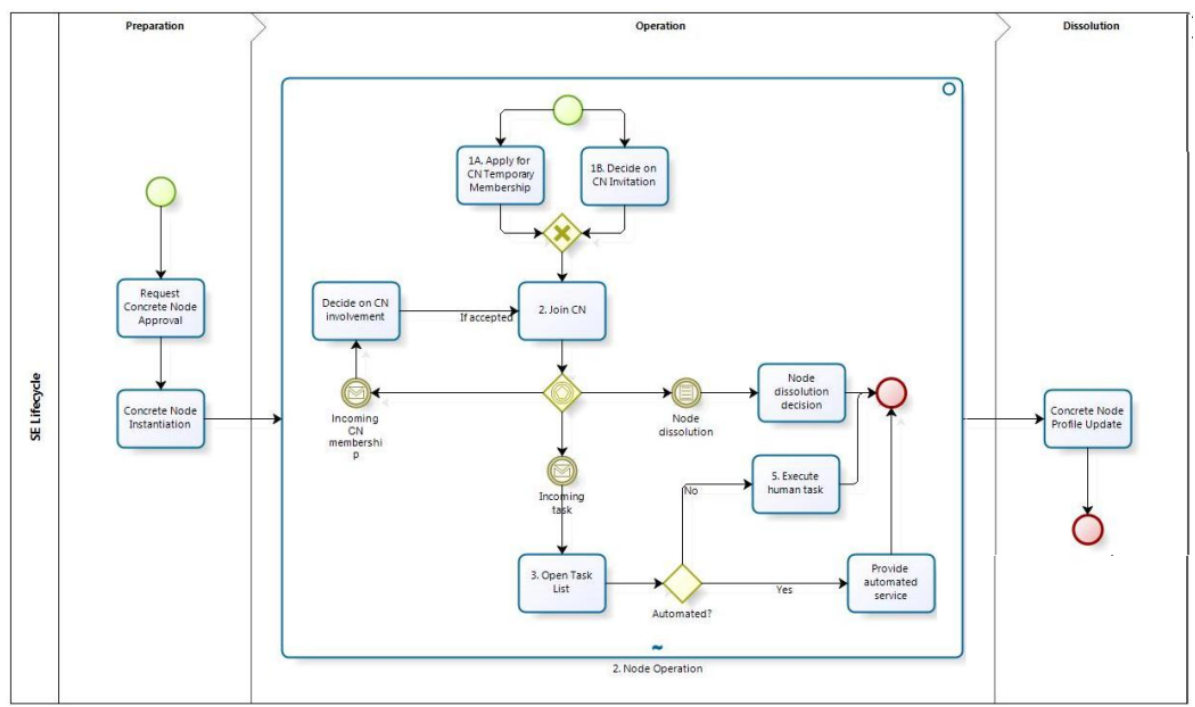

Fig. 3. Main processes at each stage of the Concrete Service Entity's lifecycle

Once they are operative, nodes may perform some specific tasks:

- Decide on invitations received from different collaborative networks. If some invitation is accepted, the node becomes part of the network and it can receive different requests from the $\mathrm{CN}$ in terms of functional contributions or transactions.

- Send an application to be considered a potential member of a CN. This mechanism goes in the opposite direction than the previous one. Here, the node offers its capabilities and services to some specific network. If accepted, it can start interacting with that network.

- $\quad$ Receive, accept and perform all the tasks coming from the network

\section{$4 \quad$ Integrated Functional Approach}

In the previous section, three lifecycles were introduced. This section will briefly explain how them are linked together in order to build up the integrated approach proposed here. Main blocks of the Fig. 4 are schematic representations of the abovedescribed lifecycles. For better comprehension, only main processes linkage is shown. 
Since the ecosystem is considered the main enabler, during its operational phase, other two lifecycles will take place:

- Service Entities: where incoming members are registered on it, and, later on, they will be ready to join future collaborative networks opportunities.

- Collaborative Networks: network managers may request the creation of a new collaborative network to the ecosystem manager by selecting the initial members from those entities already registered into it.

After the open ecosystem is created and configured, its management phase is launched. This comprises, among many others, two main activities: memberships' management and collaborative network management support.

When the creation of a new $\mathrm{CN}$ is decided, network managers will request its approval to the ecosystem manager. Once approved, the $\mathrm{CN}$ will be operative.

The operational phase of the network has many activities. For illustrating this approach, just two main processes have been included: managing the network topology, i.e. nodes being part of it; and also defining the business processes or current networked operations.

While at the same time, organizations have also started their registration process into the ecosystem. Once it is completed, they become full members of the ecosystem and they move to the network participation phase.

At this functional level, the management of the network topology is aligned with the process of organizational adhesion to a single $\mathrm{CN}$. This interaction may occur as many times as the company is either invited to join a $\mathrm{CN}$ or offering its services to it.

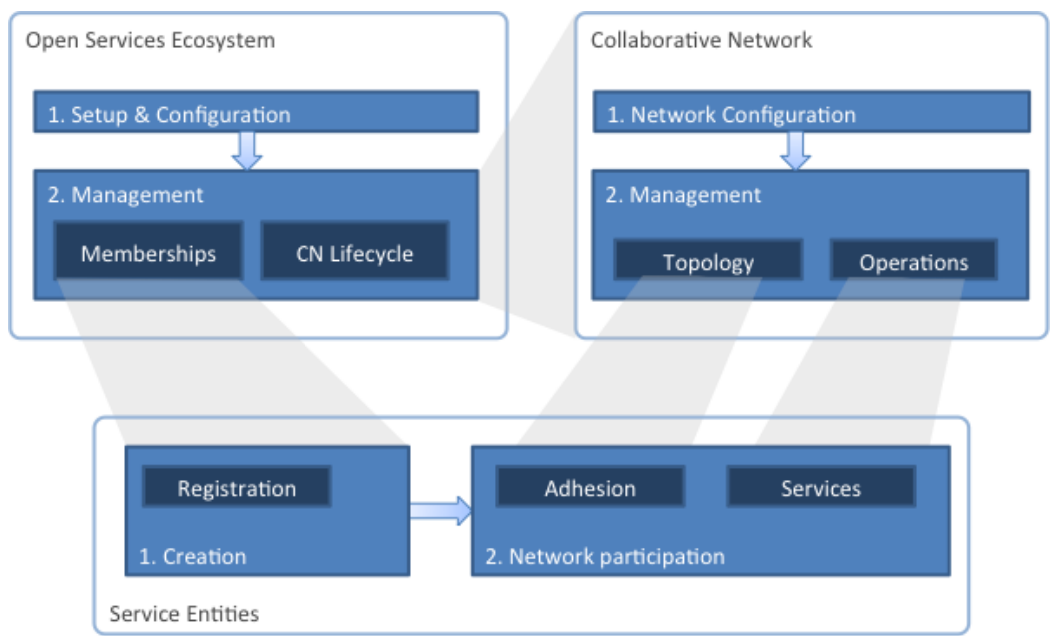

Fig. 4. Functional integration of lifecycles

Finally, when the $\mathrm{CN}$ is operative, single organizations are asked to provide their services, either manual or automated, in order to realise different extended business process instances. 
The approach proposed here considers that such expected integration can only be achieved if also the involved entities - ecosystems, collaborative networks and organizations - share a common integrated modelling framework.

\section{$5 \quad$ Integrated Modeling Views}

In the previous section, the integrated approach has been illustrated from a functional perspective. This section now describes how such integration will be realized by means of an integrated modeling framework where all the corresponding modeling views for VBE, CN and SE are properly aligned.

In the ARCON [11] reference modelling framework for $\mathrm{CN}$, a set of modelling perspectives has been proposed for their modelling.

Moreover, a modelling framework for service-based ecosystems, which identified a set of common modelling perspectives for both $\mathrm{VBE}$ and $\mathrm{CN}$ has been also proposed in [12]. The considered set of modelling perspectives includes: Processes, Services, Information/Data, Resources, Organization and Performance (see Fig. 5).

The proposed integrated approach aligns VBE/Ecosystem and CN modeling views while it also shows how Service Entities, either abstract or concrete ones, are used as integration mechanism providing consistence to $\mathrm{VBE}$ and $\mathrm{CN}$ modeling activities.

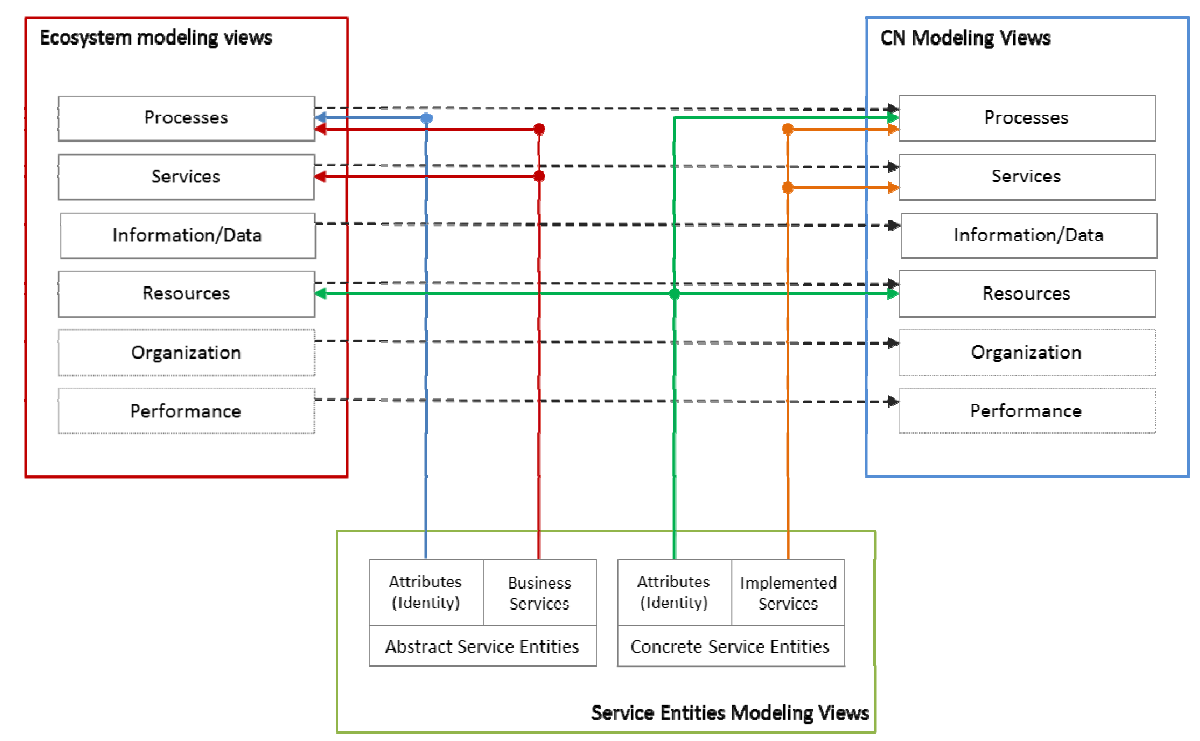

Fig. 5. Integration of modeling perspectives for Ecosystems, $\mathrm{CN}$ and Service Entities

\subsection{Using Abstract Service Entities in the Integrated Modeling Approach}

Abstract Service Entities are composed of two integrated sets that define their structure: a defined set of attributes and a set of business service interfaces. 
Attributes will be used to univocally distinguish among all its corresponding instances created while the ecosystem is operating. For the integration purposes stated here, these attributes will include, for instance, a unique name for them. This attribute will allow $\mathrm{CN}$ modellers to use it as actors of reference processes of the ecosystem.

As it, the ecosystem would include a set of reference processes which later on can be used to be instantiated for creating different $\mathrm{CN}$. Those processes would use generic references to nodes (actors) as ASE allows. Then, a reference process may include a Manufacturer, Component Supplier and Raw Material Supplier as actors of those generic processes.

When a reference process is imported into a specific CN, the ASE can be replaced by actual CSE belonging to that $\mathrm{CN}$ and which have been created by instantiating the corresponding ASE.

On the other hand, business services interfaces represent functional capabilities that each $\mathrm{SE}$ is able to provide to compose business processes. In the case of ASE, service interfaces must be considered as service specifications that, later on, concrete nodes will provide to a $\mathrm{CN}$. When used at ecosystem level, service interfaces can be used as functional parts of the reference processes. Once reference processes are instantiated into specific CNs, these business services will be implemented by actual CSE to realize process instances.

In this way, all the services defined for the ASEs are grouped into the corresponding Ecosystem y CN Service modeling view.

\subsection{Using Concrete Service Entities in the Integrated Modeling Approach}

Concrete Service Entities are instances of actual ASE defined at the ecosystem level. From the integrated modelling approach introduced here, CSE are integrated as described.

- Ecosystem/Resources: once instantiated, CSE become resources of the ecosystem. The attributes will characterize each single entity inside the ecosystem.

- CN/Resources: all the CSE registered at the ecosystem become potential nodes of a CN. The network manager will decide on the final CN structure and actual CSE will be picked up to create a new $\mathrm{CN}$.

- CN/Processes: CSE become actors of actual CN processes. Moreover, concrete services (implemented ones) will allow realizing actual extended processes instances inside the $\mathrm{CN}$.

- CN/Services: this view is formed from all the services provided by the current CSE belonging to a specific $\mathrm{CN}$.

\section{Conclusions}

This paper has introduced an approach for integrating the modeling needs arising when a functional alignment of VBE or Open Ecosystems, Collaborative Networks and Services Entities is expected.

The proposal is based on two main pillars: a) the functional alignment of the corresponding lifecycles and their adoption as main functional requirements to design 
and implement an integrated management system for them, and b) the identification and alignment of their corresponding modeling perspectives by including the Service Entities approach as a main enabler of such integration.

Thus, the ColNet platform, not focus of this work, is the result of creating an integrated system, supporting distributed business processes design and execution in collaborative networks in the context of a service-based open ecosystem.

The ColNet prototype can be accessed at www.spr.upv.es/ColNet and next research steps will include additional use cases for fully validating the overall approach.

\section{References}

[1] Osório, A.L., Afsarmanesh, H., Camarinha-Matos, L.M.: Open Services Ecosystem Supporting Collaborative Networks. In: Ortiz, Á., Franco, R.D., Gasquet, P.G. (eds.) BASYS 2010. IFIP AICT, vol. 322, pp. 80-91. Springer, Heidelberg (2010)

[2] Camarinha-Matos, L.M., Afsarmanesh, H., Ollus, M.: Ecolead: A Holistic Approach to Creation and Management of Dynamic Virtual Organizations. In: Collaborative Networks and Their Breeding Environments, pp. 3-16 (2005)

[3] ECOLEAD D22.1, ECOLEAD D22.1 VBE Management System (VMS) Requirements and Architecture Design. ECOLEAD Project WP2 (2005)

[4] ECOLEAD D23.1, Requirements and mechanisms for VO planning and launching (2005)

[5] Franco, R., Bas, Á.O., Lario Esteban, F.: Modeling extended manufacturing processes with service-oriented entities. Service Business 3(1), 31-50 (2009)

[6] Franco, R.D., Bas, Á.O., Prats, G., Varela, R.N.: Supporting Structural and Functional Collaborative Networked Organizations Modeling with Service Entities. In: CamarinhaMatos, L.M., Paraskakis, I., Afsarmanesh, H. (eds.) PRO-VE 2009. IFIP AICT, vol. 307, pp. 547-554. Springer, Heidelberg (2009)

[7] Jagdev, H.S., Thoben, K.-D.: Anatomy of enterprise collaborations. Production Planning \& Control 12(5), 437-451 (2001)

[8] Camarinha-Matos, L.M., Afsarmanesh, H.: Collaborative networks: a new scientific discipline. Journal of Intelligent Manufacturing 16(4), 439-452 (2005)

[9] Ruggaber, R.: ATHENA-Advanced Technologies for Interoperability of Heterogeneous Enterprise Networks and their Applications. Interoperability of Enterprise Software and Applications (2006)

[10] Romero, D., Molina, A.: VO breeding environments \& virtual organizations integral business process management framework. Information Systems Frontiers 11(5), 569-597 (2009)

[11] Camarinha-Matos, L.M., Afsarmanesh, H.: Towards a Reference Model for Collaborative Networked Organizations. In: Shen, W. (ed.) Information Technology For Balanced Manufacturing Systems. IFIP, vol. 220, pp. 193-202. Springer, Boston (2006)

[12] Franco, R.D., Ortiz, Á.O., Gómez-Gasquet, P., Varela, R.N.: Towards a Modeling Framework for Service-Oriented Digital Ecosystems. In: Camarinha-Matos, L.M., Boucher, X., Afsarmanesh, H. (eds.) PRO-VE 2010. IFIP AICT, vol. 336, pp. 486-493. Springer, Heidelberg (2010) 